\title{
Dinámicas excluyentes de la homosexualidad: revisión documental
}

\author{
Excluding dynamics of homosexuality: A documentary review
}

* Beatríz Collazos

** Carolina Carmona

*** Mireya Ospina Botero

\section{Resumen}

La presente revisión documental se realizó con el objetivo de indagar los factores que pueden promover las dinámicas de inclusión y exclusión de la homosexualidad en América Latina, específicamente en Colombia. Esta indagación se elaboró por medio de la búsqueda de investigaciones que tenían a modo de finalidad observar los impactos generados en los diferentes contextos en los cuales está presente.

En un primer momento, se abordará la sexualidad entendida como una base fundamental del ser humano que se encuentra atravesada por los discursos de poder, al tiempo que se contempla la noción del sistema sexo/género basado en la postura de algunos reconocidos autores que evalúan dicho concepto desde las construcciones simbólicas enmarcadas por la atribución de roles y espacios. Posteriormente, se inició una discusión con relación a los acontecimientos que han marcado el inicio y el desarrollo de la homosexualidad en el transcurso de la historia, hasta finalmente llegar a la sociedad colombiana y cómo esta percibe la "diferencia" ante las creencias que se han fabricado desde el imaginario colectivo de la normatividad.

Palabras clave: Diversidad sexual, sociedad, inclusión, exclusión.

\section{0 sumário}

A presente revisão documental é realizada com o objetivo de investigar os fatores que podem promover os discursos de inclusão e

* Estudiante de la Fundación Universitaria Católica Lumen Gentium, cursando décimo semestre postulada al título de psicóloga. Monitora de investigación del proyecto "Configuración de familia, maternidad y paternidad en estudiantes de Unicatólica 2017" Monitora de investigación en el proyecto familia y diversidad sexual en estudiantes universitarios en la primera parte de la misma para el año 2017. Auxiliar de la investigación "familia y diversidad sexual en estudiantes universitarios" como modalidad de grado y práctica profesional 2018. Contacto: collazosbeatriz5@gmail.com

** Docente Investigadora Fundación Universitaria Cátolica Lumen Gentium Cali. Grupo de Investigación Lumen Humanitas. Psicóloga Universidad Javeriana Cali, Magister en Familia. Universidad Javeriana Cali. Contacto:carolinacarmonac3@gmail.com.

** Docente Investigadora Universidad Católica de Pereira. Psicóloga de la Unad. Especialista en Psicoterapia Sistemática, de la Universidad de Manizales. Magister en Educación y Desarrollo Humano. Fundación Centro Internacional de Educación y de Desarrollo Humano. Email: mireya.ospina@ucp.edu.co 
exclusão sobre diversidade sexual na América Latina especificamente na Colômbia, investigando através da busca de pesquisas cujo objetivo é falar sobre diversidade e os impactos que podem ser gerados nos diferentes contextos em que está imerso.

A princípio, a sexualidade entendida como base fundamental do ser humano que é atravessada pelos discursos de poder, contemplando a noção do sistema sexo / gênero a partir da posição de alguns autores reconhecidos que avaliam o conceito das construções simbólicas emolduradas pela atribuição de papéis e espaços. Posteriormente, iniciou-se uma discussão sobre os eventos que marcaram o início e o desenvolvimento da homossexualidade no curso da história, até finalmente chegar à sociedade colombiana e como ela percebe a "diferença" antes das crenças que foram fabricadas desde imaginário coletivo da heteronormalidade.

Palavras chave: Diversidade sexual, sociedade, inclusão, exclusão.

Actualmente, hablar sobre diversidad sexual implica empezar a generar consciencia sobre las limitadas posibilidades que le son otorgadas a personas que hacen parte de la diversidad sexual en una sociedad heteronormativa que induce a la censura de quienes son diferentes y limita la libertad de expresión (Wittig, 1971).

Esta situación ha despertado el interés para empezar a cuestionar vivencias de vulnerabilidad como la violencia física, verbal, psicológica e incluso económica que da inicio en el hogar y se extiende hasta los contextos laborales y sociales (Lozano, 2009; Barrios, 2018) que atraviesan los miembros de la comunidad LGBT (Lesbianas, Gays, Bisexual, Transexual) en Colombia. Es por esto, que desde la Fundación Universitaria Católica Lumen Gentium surge la necesidad de conocer las experiencias que viven las personas con diversas orientaciones sexuales y de género, por lo que se propuso llevar a cabo una investigación cuyo objetivo era comprender los significados y vínculos alrededor de la diversidad sexual en los estudiantes LGBT de las universidades católicas de Pereira, Lumen Gentium y sus familias. Durante el desarrollo de este proyecto fue posible observar los frecuentes señalamientos sociales de los cuales son víctimas y es precisamente teniendo en cuenta los relatos de dichos participantes que se hizo imperativo iniciar una búsqueda documental que permitiera ayudar a comprender algunos de los posibles factores que influencian las conductas de rechazo hacia la población sexualmente diversa. Consulta llevada a cabo en bases de datos como Dialnet, Doaj, Gale Cengage Learning, utilizando las palabras claves las cuales arrojaron resultados de 700 documentos aproximadamente entre investigaciones, tesis y artículos útiles para esta revisión un $8 \%$ de ellos.

- La sexualidad entre las dinámicas de poder

Para Foucault (1976), la sexualidad es el resultado de la réplica de dinámicas de poder que sobresalen dentro del discurso social en donde se vigila, en primer lugar, la experimentación del acto sexual desde las fantasías o los deseos e inclusive el placer y en segundo lugar, se regula la natalidad de la especie, lo que quiere decir que dicha sexualidad no corresponde a una expresión innata del ser humano, sino más bien a los aprendizajes adquiridos en la interacción histórica de la sociedad y la necesidad de la misma por dominar mediante el lenguaje social implícito.

Es por esto que, aunque las personas consideren que su definición de sexualidad se construye teniendo en cuenta los roles asignados según el género y a las concepciones personales que el sujeto ha realizado a lo largo de su vida, no ocurre así. Por el contrario, siempre se encuentra presente la influencia de la sociedad, frente a esto Figari (2007) señala que "la cultura establece las restricciones del "cómo" [...]" (p. 20) y la sociedad señala "con quien". Lo que quiere 
decir que la sexualidad de la persona es única para cada individuo y se ve modificada por la influencia de los procesos diarios y las reglas del contexto en el que está inmerso (Vidal, 2002; Olivera, 2009).

De tal forma, Wittig (1992) y Beasley (2006) afirman que la sexualidad es vista como el resultado de las estructuras patriarcales en la búsqueda de agrupar o encasillar al ser humano en las categorías de heterosexual y homosexual, facilitando la polarización, el señalamiento y la exclusión de aquellos individuos que no actúan conforme a las demandas sociales.

Precisamente Kiernan (citado por Katz, 2007) acuña el término de heterosexualidad y lo define como la atracción sexual y emocional hacia el sexo opuesto, por lo que en efecto Wittig (1971) propone que esta categoría es la forma más eficiente de asegurar los mecanismos de dominación colectiva, dado que la heterosexualidad es elemental para dar sentido a la sociedad, mientras ordena las relaciones humanas.

Sucesivamente, la homosexualidad, según Cesari (1998), es la atracción o el deseo sexual y afectivo entre sujetos del mismo sexo; a lo que Beasley (2006) declara que al crear dichas categorías surge la intolerancia y las conductas discriminatorias que han generado el marginamiento, exclusión, rechazo e incluso la muerte por la forma de vivenciar la sexualidad y desafiar a la norma (Chávez, Zapata, Petrzelova, Villanueva, 2018). En la medida en que las dinámicas de poder sienten la necesidad de dominar, crean la sexualidad para clasificar las conductas sexuales y agrupar a los seres humanos en las categorías de heterosexualidad y homosexualidad que se apoyan en los roles asignados a cada género (Beasley, 2006).

\section{Conceptualizando el término}

\section{sexo/género}

A partir de unas primeras aproximaciones a la categoría de género, Lamas (2000) menciona que este concepto debe entenderse teniendo en cuenta factores biológicos y sociales, debido a que el género es la construcción cultural utilizada para delimitar las diferencias sexuales. Es decir, que el género se encarga de marcar los posibles roles que son atribuidos a cada sexo, a partir de una construcción simbólica que, a su vez, se convierte en el punto de partida para nuevas dinámicas sociales particulares y subjetivas, las características atribuidas como femeninas o masculinas son resultado de un proceso tanto en el ámbito individual como en la relación con el medio social; es decir, este género no se adquiere de forma innata, sino que, por el contrario, se obtiene a través de la interacción social en la cual existen algunas concepciones atravesadas por lo político y lo cultural, donde este concepto podría distar de lo socialmente establecido.

De este modo, Rubín (1986) expone la existencia de un sistema sexo/género, que no es innato al igual que la sexualidad y se ha encargado de transformar en una obra de producción a la humanidad. Este mecanismo se encarga de modificar a machos y hembras en hombres o mujeres adaptados a una sociedad particular, enmarcada por la desigualdad de los géneros a partir de las diferencias entre los sexos. Coincidiendo con Thurén (1993) en que el sistema sexo/género incluye diversas formas de someter a la población, mediante la distribución del trabajo y la jerarquización de las funciones, encasillando a lo femenino en el ámbito privado (el hogar, la crianza, el cuidado, servicio u obediencia, la reproducción) y a lo masculino en el ámbito público (remuneración, movilidad, interacción, poder). Por ejemplo, dentro del ámbito laboral aún se excluye frecuentemente a la población diversa, negándose la posibilidad de desarrollarse y ser reconocidos en el ámbito profesional (Burgos y Turriago, 2016).

Así es como el poder que enmarca la sexualidad puede también clasificar las conductas, comportamientos y actitudes asignados a cada género según las características sexuales anatómicas, prolongando el dominio de la huma- 
nidad por parte de la masculinidad, mediante los discursos heteronormativos latentes en las relaciones sociales que se transmiten por medio de los sistemas de creencias y se han convertido en una herramienta para continuar cosificando a las personas (Rubín, 1986). Ahora bien, es el momento indicado para hablar sobre los sistemas de creencias definidos por Dallos (1996) como un conjunto de interpretaciones que perdura en el tiempo sobre lo que se considera auténtico sin necesidad de ser verificados. Al ser transmitido por el sistema familiar y el contexto social, los sistemas de creencias son la base fundamental para esta revisión.

Lo que representa un factor incidente en el rechazo o inclusión de la comunidad LGBT, como se evidencia en el siguiente recorrido histórico en donde convergen los acontecimientos más significativos que ilustran la lucha por la reivindicación de los derechos para quienes hacen parte de la diversidad sexual.

\section{La homosexualidad: Hitos}

Este recorrido histórico inicia con los postulados de Halperin (2000) sobre la actividad sexual de los hombres en la Grecia clásica y en la antigua Roma, en donde las mujeres se encargaban únicamente de las funciones del hogar y la relación heterosexual era utilizada para sostener la especie, pero en esta medida el hombre solo lograba alcanzar la satisfacción sexual por medio de las relaciones entre sujetos del mismo sexo. Es importante resaltar que aquel hombre que tuviese mayor poder era quien tenía control en la relación sexual, fuese con hombres o mujeres. Lo anterior se asume a través de su filosofía, aceptando cualquier tipo de deseo con un fin hedonista.

Para el siglo XVII se da inicio a la clasificación de las conductas sexuales como enfermedades y es en este momento donde aparece el término desviación sexual que correspondía a cualquier acto que no tuviese un fin meramente reproductivo, dado que estos afectan la salud física y psicológica de los sujetos; la homosexualidad no ingresa a esta categorización sino hasta el siglo XIX (Boswell, 1998). Para esta época se plantea el modelo isomorfico definido por Galeno citado en Foucault (1991), como las semejanzas entre los aparatos anatómicos de hombres y mujeres los cuales se diferencian en la ubicación del humor en la mujer, haciendo referencia a que la mujer era un hombre incompleto, lo que es refutado por Laqueur (1994), al estudiar los cuerpos del ser humano y encontrar la existencia de diferencias entre los órganos sexuales de las mujeres y de los hombres, es decir, dos cuerpos completamente distintos que, para el momento, no eran considerados como tales a lo que llamó dimorfismo sexual. Con esta diferenciación es que se han catalogado y estructurado las relaciones de sexo/ género en la actualidad.

Continuando en el siglo XIX, esta vez ubicados en Alemania, se hallan los pioneros del movimiento LGBT, entre ellos Karl Ulrichs quien propone la teoría del uranismo desde el deseo o una perspectiva de diversos tipos de amor llamándolo el tercer sexo. Frente a esto, Ulrichs luchó por los derechos de los uranistas e incluso fue la primera persona en declararse públicamente uranista. Poco tiempo después, en el año de 1868, el austriaco Kertbeny dentro de sus cartas introduce el término homosexual y heterosexual (Routledge, 1997).

Durante el año 1931, en Alemania, se efectúan los primeros tratamientos hormonales y posterior reasignación de sexo a Lili Elbe, quien muere después de realizarse una precipitada cirugía de reasignación de sexo (Beltrán, 2003).

Uno de los más grandes acontecimientos para la comunidad LGBT durante este siglo se presentó el 28 de junio de 1969 en los Estados 
Unidos, denominado los disturbios de Stonewall, que surgieron como una manifestación de mujeres transexuales contra una redada policial, que irrumpió en bar de la ciudad de Nueva York conocido como Stonewall Inn. Frente a este suceso, dos años después, en la misma fecha, se empezaron a formar grupos de hombres y mujeres del Frente de Liberación Gay (GLF) en diversas ciudades como Filadelfia y Nueva York para recordar este hecho, lo que no imaginaron fue que más tarde se convertiría en el día internacional del orgullo LGBT.

De acuerdo con lo anterior, para el año de 1990, la Organización Mundial de la Salud retira la homosexualidad de su lista de enfermedades mentales, de esta forma se abrió el panorama en torno a la subjetividad, tratando de aceptar el punto de vista que tiene el otro sobre su propia sexualidad (Ben, 1997).

Rodríguez (2008) realiza una recopilación de los acontecimientos más significativos alrededor del mundo, donde se legalizaron algunos derechos fundamentales para la comunidad LGBT como: En 1974 se inaugura la primera línea de atención telefónica para la población diversa sexualmente llamada "London Lesbian and Gay Switchboard" y se realiza por primera vez una conferencia mundial de derechos LGBT en Edimburgo, Escocia.

Durante el año 1998, Holanda sobresale como el primer país en legalizar el matrimonio entre personas del mismo sexo y para el 2011, se valida la adopción. Bélgica, en el 2003, incursiona legalmente en este ámbito y decreta en su código civil la legalización de esta unión. Durante el año 2005, Canadá y España se unen a los países donde se ha legalizado el matrimonio entre personas del mismo sexo. En esta misma línea se resaltan países como Argentina, Finlandia e Islandia, entre otros 19 países donde se permite el matrimonio homosexual (Veiga, 2017).

Aun así, más de 72 países siguen prohibiendo o restringiendo la unión entre parejas del mismo sexo y en América se ubican diez de ellos que continúan sin tener leyes que promuevan los derechos para personas diversas sexualmente como Barbados y Guyana (Clarín, 2017). Incluso en Brasil se cometen crímenes por motivos de odio hacia la comunidad LGBT cada 19 horas (Alves, 2018). Frente a estos datos se revisan estudios acerca de la percepción que tiene la población de América Latina sobre la diversidad sexual y de este modo comprender cuáles pueden ser los factores que influencian los comportamientos o actitudes de inclusión o exclusión de la comunidad LGBT.

\section{Algunas investigaciones en América Latina}

En las últimas décadas, la situación de discriminación y exclusión que vivencia la población LGBT continúa en aumento, a pesar de la lucha por la inclusión social y laboral por la que han luchado a lo largo de la historia.

Un trabajo realizado por Gómez y Barrientos (2012) en Chile, da cuenta que se están promoviendo discursos discriminatorios acerca de la diversidad sexual en espacios públicos, sobre todo por los prejuicios asociados a los homosexuales, mientras que Barrientos y Páez (2000), King (2001), Bowen y Bourgeois (2001) señalan que dichos comportamientos y actitudes están relacionadas con ubicación demográfica y el estatus financiero de la familia. Aunque Asencio (2006) manifiesta que estas actitudes discriminatorias se relacionan con la nula interacción con la diversidad, junto con las concepciones negativas alrededor de la comunidad LGBT existentes dentro del núcleo familiar y el entorno social cercano.

Por otra parte, Lozano (2009), en México, identifica la persistencia de patrones y estereotipos machistas en donde las expresiones de diversidad sexual son discriminadas en su totalidad con violencia verbal, física y psicológica desde el hogar. Representando vergüenza para el grupo familiar y sentimientos de culpa en aquel sujeto con una 
orientación sexual diversa (Díaz, 2004). Estas creencias se han consolidado en el interior de la población, a través de un proceso que ordena las identidades de la sociedad y justifica los discursos discriminatorios, la aceptación de subordinación y de injusticia (Morales, 2014).

En esta misma línea, Moral de la Rubia, Valle de la O. y Gómez. (2013) realizaron un estudio en ciudad de Monterrey donde identificaron el nivel de rechazo en 221 estudiantes de medicina y psicología. Entre sus resultados más importantes se evidencia que al menos un cuarto de los participantes aún vulnera o rechaza a las personas por su condición sexual y esta discriminación es más fuerte hacia las lesbianas.

En Ecuador, Pinos y Pinos (2011) identificaron factores de predisposición como: el tipo de colegio, la etapa de la adolescencia, el sexo, alto nivel de religiosidad, baja escolaridad de padre y madre, prejuicios hacia la comunidad LGBTI que se ven relacionados con las actitudes negativas hacia la homosexualidad. Con respecto a lo anterior, hay una particularidad y es que son los hombres quienes discriminan en mayor medida a parejas de hombres. Coincidiendo con la investigación en Puerto Rico, Argentina, México y Colombia, en donde las creencias religiosas, la baja escolaridad y la presencia marcada del patriarcado se convierten en factores que defienden la exclusión de la homosexualidad (Fernandez, 2013; Moral de la Rubia; Valle de la O. y Gómez, 2013; Frenkel-Brunswik, Levinson y Sanford, 1950).

Adicionalmente, Alfonso y Díaz (2004) identifican que tal vez la poca apertura hacia el exterior incide en la discriminación, como lo muestran en su investigación acerca de la forma en que se vive la exclusión desde las universidades de San Juan en Puerto Rico, por medio de una investigación de carácter cuantitativa, que contó con la participación de 548 estudiantes, encontrando mayor prejuicio en hombres hacia hombres, debido a la heteronormatividad y a los estereotipos que sobresalen al interactuar con miembros de la comunidad LGBT.

Buitrago y Pérez (2017) realizan una revisión documental de 30 investigaciones realizadas en América Latina en donde logran identificar percepciones, en su mayoría negativas hacia la comunidad LGBT, además de dificultades para aprobar las relaciones homosexuales por desviarse de la línea heterosexual, más aún porque se conciben como un hecho meramente sexual que no conlleva a un vínculo más fuerte, directamente relacionado con las creencias culturales transmitidas generacionalmente por la población tradicional y conservadora.

Las anteriores investigaciones resaltan que la discriminación y la exclusión de la diversidad sexual se presenta por la baja escolaridad, el poco conocimiento acerca de la diversidad sexual, los patrones culturales conservadores, las concepciones negativas, los estereotipos y prejuicios, todos estos factores se ven ligados con los sistemas de creencias sociales que Dallos (1996) define como un conjunto de interpretaciones que perdura en el tiempo sobre lo que se considera auténtico, según el grupo familiar y el contexto social que es transmitido de generación en generación.

Así es como los discursos negativos que desnaturalizan, se realizan con el objetivo de ratificar y amparar las creencias sociales que desde la heteronormalidad escenifican el factor número uno sobre los discursos de inclusión o exclusión hacia la población diversa, por medio de estereotipos y prejuicios que generalizan la forma de percibir al mundo, justificando el señalamiento, la burla y la violencia hacia aquellos que no actúan conforme a los lineamientos sociales.

\section{¿Colombia, tierra de todos?}

Durante los años 40, se forma el primer grupo de hombres gay de clase alta en el país, conocido como "los felipitos" que buscaba ge- 
nerar espacios de homosocialización. Poco después, este grupo desapareció sin dejar rastro alguno de sus miembros (Acevedo, 2013; Rodrigez, 2017). La despenalización de la homosexualidad se efectúa en el año de 1981, gracias a esta medida surgieron movimientos colectivos como Grupo de Estudio y Liberación y el Movimiento por la Liberación Homosexual. Durante el transcurso del siguiente año se realiza la primera marcha del orgullo gay en Bogotá. (Turizo e Iglesias, 2010). Esta es una de las formas en que la comunidad LGBT trató de visualizar y buscar la reivindicación de sus derechos.

Colombia, actualmente, es uno de los países de América Latina que acepta las relaciones entre personas del mismo sexo, permite el matrimonio igualitario e hizo legal la adopción para el año 2015, incluso es posible efectuar el cambio de nombre, la reasignación de sexo o modificaciones corporales sin un permiso judicial. Aun así, la comunidad LGBT en el país está regida por un panorama de señalamientos discriminatorios y excluyentes que se inician en el núcleo familiar y se extiende en los espacios sociales, frente a la dominación de una cultura que señala a quienes deciden no seguir con los parámetros socialmente establecidos, como lo resaltan Fernández, Gutiérrez y Quijano (2013) en la investigación realizada en la Universidad del Valle de Santiago de Cali, acerca de las representaciones sociales de la homosexualidad en estudiantes, revelando que la religión (Grajales, Ossa, Klimenko y Posada, 2015) influencia las percepciones negativas frente a la homosexualidad y junto con las cátedras educativas legitima las creencias sociales discriminatorias.

De acuerdo con lo anterior, es frecuente encontrar casos de violencia contra personas diversas sexualmente, como, por ejemplo, (Barrios, 2018), Santiago de Cali es la ciudad con mayor expresión de violencia contra la comunidad LGBT, de este modo, para el año 2017 se presentaron 109 asesinatos con motivos de pre- juicio en donde los mayores afectados son los gays y las mujeres trans (Agencia EFE, 2018). No obstante, se han reducido las tasas de muertes violentas por motivos de odio en el país, aunque los casos existentes quedan en impunidad, esto sitúa a la población diversa en un mayor grado de vulnerabilidad (Cantor, 2009), es decir, que socialmente el avance hacia la aceptación e inclusión de la diversidad sexual ha sido muy lento, puesto que los discursos que señalan, rechazan y minimizan al otro promueven la discriminación. Quitian y Prieto (2011) estudian los discursos de alumnos y profesores de la Pontificia Universidad Javeriana de la ciudad de Bogotá, en búsqueda de determinar las concepciones sobre la diversidad sexual, identificando que para los participantes la diversidad sexual corresponde a una forma diferente de llevar la sexualidad, pero es un tema que aún representa un tabú para la sociedad ante la falta de información y reconocimiento, persistiendo las tendencias discriminatorias que hacen más tardía la inclusión de la comunidad LGBT. De la misma manera, Castelar y Quintero-Aguirre (2012) analizan los discursos de odio en siete hombres homosexuales de la ciudad de Cali, en donde los participantes revelan la necesidad de ocultar su expresión de género para ser aceptados socialmente, dado que al tener comportamientos que corresponden al género femenino pueden llegar a ser objeto de amenazas, burlas, ofensas y agresiones físicas.

Burgos y Turriago (2016), por medio de su investigación/intervención, indagaron por las narrativas de inclusión y exclusión en el entorno laboral, logrando reconocer que los prejuicios y estereotipos son imaginarios que se han instaurado en la sociedad desde contextos laborales, sociales, familiares y religiosos; limitando o hasta ignorando los recursos y potenciales de las personas por su diversidad.

\section{Discusión}

El desarrollo de esta revisión documental permitió reconocer que la sexualidad es una herramienta 
creada y utilizada por el patriarcado para controlar a los seres humanos, siendo naturalizada a través de las relaciones sociales a lo largo de la historia, junto con los roles y espacios asignados a cada sistema de sexo/genero, clasificando las conductas sexuales en la heterosexualidad (la normal, lo que es correcto) y la homosexualidad (anormalidad), grupos opuestos delimitados entre el bien/el mal, el cómo, el cuándo y con quién es correcto experimentar la sexualidad de acuerdo con la percepción de una sociedad particular, dirigida por los lineamientos de poder que con la ayuda del lenguaje han trascendido hasta la actualidad, inmersos entre los sistemas de creencias colectivos, factor elemental en los discursos, señalamientos y comportamientos de discriminación contra la diversidad (Foucault, 1976; Rubín, 1986; Dallos, 1996).

Frente a este panorama cabe resaltar que la sociedad colombiana está relacionada con la cultura de México, Puerto Rico, Argentina, Chile y Ecuador, entre algunos de los países latinos con quien Colombia comparte sus raíces, en la medida en que sus creencias sobre la diversidad se impulsan desde un contexto patriarcal/hegemónico que (Burgos y Turriago, 2016; Garzón y Seoane, 1992) legítima discursos que promueven actitudes y comportamientos excluyentes e incluso factores como la inclinación política, la edad, la religión, la cultura, el nivel educativo, los estereotipos, el desconocimiento y los prejuicios corresponden a la forma en que se percibe la realidad ante la construcción e interiorización de creencias que le dan un sentido y un significado negativo a la homosexualidad, propio del contexto en que se está inmerso (Frenkel-Brunswik, Levinson y Sanford, 1950), es decir, son los sistemas de creencias los que perduran en el tiempo y promueven las actitudes, comportamientos y discursos negativos contra la diversidad sexual que pueden ser modificados en la misma medida en que son instaurados.

La mayoría de los estudios que se consultaron dan cuenta de que se puede convivir con la diversidad sexual, siempre y cuando no se hagan demostraciones afectivas en lugares públicos o las parejas se reúnan en espacios familiares, lo que representa discriminación hacia las parejas del mismo sexo. En particular, se resalta que son los hombres quienes discriminan en mayor medida a parejas de hombres, manifestando repulsión sobre las muestras de afecto. Por lo cual, es evidente encontrar entre el discurso de los jóvenes diversos, una latente sensación de rechazo y discriminación en su entorno, aún más cuando sus comportamientos corresponden a lo femenino, (Barrientos y Páez, 2000; King, 2001; Bowen y Bourgeois, 2001) puesto que los reproches, insultos y cualquier otra forma violencia no se hacen esperar. Continuando por esa misma línea, se reconoce menor comprensión por parte de familiares de chicas transgenero/ transexual, siendo las víctimas más frecuentes de violencia al interior del hogar (Castelar y Quintero-Aguirre, 2012) ante la creencia de perder aquellos privilegios que el hombre, por nacer hombre, ha ganado ante las relaciones de poder que se han gestado en el interior del sistema social (Scott, 1996).

Estos son comportamientos y actitudes relacionadas con los patrones heteronormativos presentes en la cultura. Sin embargo, se encuentra que las mismas expresiones en las mujeres pueden llegar a aceptarse con mayor facilidad incluso desde el ámbito religioso (Díaz, 2004). Lo que demuestra contradicciones ante la relativa inclusión que se hace a un grupo específico que compone la diversidad sexual, correspondiente a la forma en que son percibidas las expresiones amorosas entre mujeres atreves de los sistemas sexo/género que cosifican lo femenino y lo convierten en un producto construido simbólicamente para satisfacción a los hombres (Alfonso, 2012; Buitrago y Pérez, 2017; Scott, 1996)

Hablar sobre la exclusión, y mucho más sobre la inclusión, resulta ser un tema bastante complejo puesto que excluir o rechazar parece ser más sencillo y es lo que corresponde, de acuerdo con los lineamientos heteronormativos, por lo cual se hace necesario redefinir las creencias planteadas desde la heterosexualidad que no permite la expresión de la diversidad del ser humano y en cambio limita al sujeto a través de la asignación de roles que estigmatizan. 
¿Cómo hacerlo? Modificar estos paradigmas en búsqueda de una sociedad más abierta y equitativa demanda el esfuerzo de todos los ciudadanos, más aún de los psicólogos mediante campañas psicoeducativas que posibilitan la información y el conocimiento a la población, puesto que es la educación la que puede transformar la sociedad, conocimientos que pueden convertirse en herramientas para la apropiación de la sexualidad en la sociedad colombiana, facilitando la interacción entre toda la comunidad sin continuar dividiendo a las personas entre heterosexuales y homosexuales, construyendo así una realidad colectiva que favorezca el contacto entre la sociedad y, en un futuro, a eliminar dichas creencias negativas.

\section{Referencias bibliográficas}

Acevedo, T. (2008). Lesbianismo y construcción del sujeto homosexual femenino en Colombia visto a través del poemario "rupturas" de fedra (tesis inédita de maestría/doctorado). Pontificia Universidad Javeriana, Bogotá, Colombia.

Agencia EFE. (Julio 05, 2018). Durante el 2017 fueron asesinadas 109 personas LGBTI en Colombia. El País, recuperado de https://www.elpais.com. co/judicial/durante-el-2017-fueron-asesinadas109-personas-lgtbi-en-colombia-revela-informe. html

Alfonso, J. (2012). El Estado Actual de la Investigación Sobre la Discriminación Sexual. Terapia psicológica, $30(2), 71-76$.

Alfonso, J. y Díaz, N. (2004). Los otros: prejuicio y distancia social hacia hombres gay y lesbianas en una muestra de estudiantes de nivel universitario. International Journal of Clinical and Health Psychology. 4(3), 537-551 Granada.

Alves, M. (03/ 01/ 2018). Radiografía de crímenes de odio en America Latina. La Nación, recuperado de https://www.lanacion.com.ar/2101891-crimenes-de-odio-en-america-latina.
Asencio, C. (2006). Percepciones sobre la diversidad sexual que tienen estudiantes de la carrera de Obstetricia y Puericultura durante su formación profesional en la Universidad Austral de Chile (tesis inédita de pregrado). Universidad Austral de Chile, Valdivia, Chile.

Barrientos, J. E., \& Páez, D. (2006). Psychosocial variables of sexual satisfaction in Chile. Journal of sex \& marital therapy, 32(5), 351-368.

Barrios, L. (2013). La población de lesbianas, gays, travestis, bisexuales e intersexuales (LGBTI) en el departamento del Atlántico. Manzana de discordia, 8 (1), 23-35.

Beasley, C. (2005), Gender y sexuality: critical theories, critical thinkers, London, SAGE.

Beltrán, S. (2003). Sexualidades migrantes Género y transgénero. Maffia, D (Compiladora). Buenos Aires: Feminaria.

Ben, P. (2014) Las relaciones sexuales entre personas del mismo sexo y el origen histórico de la homosexualidad. Razón y Revolución, 3, 1-17.

Boswell, J. (1993). Cristianismo, Tolerancia Social y Homosexualidad. Los gays en Europa occidental. Muchnik. Barcelona.

Bowen, A. y Bourgeois, M. (2001). Attitudes toward lesbian, gay, and bisexual college students: The contribution of pluralistic ignorance, dynamic social impact, and contact theories. Journal of American College Health, 50, 91-96

Buitrago, F. y Pérez, A. (2017). Actitudes frente a la homosexualidad en latinoamérica (tesis inédita de maestría/doctorado). Universidad Cooperativa de Colombia, Bucaramanga, Colombia.

Burgos, L. Y Turriago, N. (2016). Narrativas de inclusión-exclusión laboral en comunidad LGBTI, familia y contextos laborales (tesis inédita de maestría). Universidad Santo Tomas, Bogotá, Colombia. 
Cantor, E. (2009). Cultura estudiantil y diversidad sexual discriminación y reconocimiento de los y las jóvenes LGBT en la secundaria. Polisemia, 8, 101110.

Carpenter, E. (1914). Intermediate Types among Primitive Folk, Allen and Unwin, Londres: Editorial.

Castelar, A., y Quintero Aguirre, F. (2012). Performatividad y lenguaje de odio: expresiones de la homosexualidad masculina en la ciudad de Cali. Revista CS, (10), 207-240.

Cesari G. (1998) "Natura ed interpretazione dell'omosessualità", en Sgreccia E., (a cura di) AIDS Problemi bioetici correlati con la clinica l' epidemiologia e le implicazioni socio-culturali della sindrome da inmunodeficienza acquisita. Bolongo 100.

Chaparro, N. Y Vargas, S. (2011). Imágenes de la diversidad. El movimiento de liberación LGTB tras el velo del cine Culturales, VII (14), 57-86.

Chávez, M., Zapata, J., Petrzelová, J. y Villanueva, G. (2018). La diversidad sexual y sus representaciones en la juventud. Psicogente, 21(39), 62-74.

Chomali, F. (2008). Algunas consideraciones para el debate actual acerca de la homosexualidad. Centro de bioética, Santiago.

Clarín (19/06/2017). Informe internacional. En estos 72 países ser gay es ilegal. Clarin. Recuperado de https:// www.clarin.com/sociedad/72-paises-gay-ilegal_0_ HyxFSDSmb.html

Dallos, R. (1996), Sistema de creencias familiares, Barcelona: Paidós.

Díaz, M. (2004). Homosexualidad y género. Cuicuilco, 11 (31), Distrito Federal.

Fernández, A., Gutiérrez, J. Y Quijano, M. (2013). Representaciones sociales sobre la homosexualidad en estudiantes heterosexuales de Psicología y de Biología: un estudio descriptivo. Teoría y crítica de la psicología 3, 40-62.
Fernández, M. (2013). Perspectivas sobre la homosexualidad en la historia reciente de Argentina. Aportes, limitaciones y enfoques Apuntes de investigación del CECYP, 23, 153-185.

Frenkel-Brunswik, E., Levinson, D. y Sanford, A., (1950). La medición de tendencias antidemocráticas implícitas. La personalidad autoritaria , 222-279

Figari, C. (2007). Sexualidad, ciencia y religión. Encuentro, Buenos Aires.

Foucault, M. (1976). Historia de la sexualidad, vol I. La voluntad del saber. México: FCE (1991). Microfísica del poder, La piqueta. Madrid.

Gamboa, L. (1998). Historia de la sexualidad. Rev Biomed, 9 (2). 116-121.

Garzón, A. y Seoane, J. (1992). Creencias sociales contemporáneas, autoritarismo y humanismo .Psicología Política, 5, 27-52.

Gilmore, D. (1994). Hacerse hombre. Concepciones Culturales de la masculinidad. Barcelona: Paidós.

Gómez, F. y Barrientos, J. (2012). Efectos del prejuicio sexual en la salud mental de gays y lesbianas, en la ciudad de Antofagasta. Sexualidad, Salud y Sociedad. 10. 100-123.

Grajales, B., Ossa, C., Klimenko, O., y Alvares, J. (2015). Percepciones de algunas personas de orientación homosexual sobre el reconocimiento de la comunidad LGTBI a nivel social en Colombia. Psicoespacios, 9 (15), 36-70.

Halperin, D. (2000). How to Do the History of Homosexuality. GLQ: A Journal of Lesbian and Gay Studies, 6 (1), 87-123.

Katz, J. (2007). The invention of heterosexuality. University of Chicago Press, Chicago.

Kennedy, H. (1997). Karl Heinrich Ulrichs First Theorist of Homosexuality. Science and homosexualities, 26, 26-45 
King, B. (2001). Ranking of stigmatization toward lesbians and their children and the influence of perception of controllability of homosexuality. Journal of Homosexuality, 41(2), 77-97.

Lamas, M. (2000) "Diferencias de sexo, género y diferencia sexual". Cuicuilco, 7 (18), 95-118.

Lombana, D. (2014). Sexualidad Y Poder En La Obra De Michel Foucault (tesis inédita de de pregrado). Universidad De Cartagena, Cartagena de Indias, Colombia.

Lozano, I. (2009). El significado de homosexualidad en jóvenes de la ciudad de México. Enseñanza e investigación en psicología, 14 (1) 153-168.

Mexico, U. (2016). La OMS elimina la homosexualidad del catálogo de enfermedades mentales.

Misitu, G. (1993) Psicología de la comunicación humana, Buenos Aires: Lumen.

Montoya, G. (2016). La invención clínica del homosexual en Colombia, 1890-1980. Revista de Psicología Universidad de Antioquia, 8(2),121-146.

Moral de la Rubia, J., Valle de la O., Gómez, E. (2013). Evaluación del rechazo hacia la homosexualidad en estudiantes de medicina y psicología con base en tres escalas conceptualmente afines. Psicología desde el caribe, 30 (3), 526- 550.

Morales, Z. (2014). Machos y machistas: Historia de los estereotipos mexicanos. La ventana. Revista de estudios de género, 5(39), 252-260.

Núñez, N. (1999). Sexo entre varones. Poder y resistencia en el campo sexual. México: Porrúa.

Pinos V. y Pinos A. (2011). Actitudes de los adolescentes escolarizados de Cuenca hacia la diversidad sexual. MASKANA, 2(1), 1-16.

Quitian, A., y Prieto, E. (2011). Análisis semántico del discurso sobre diversidad sexual y sobre la politica pública LGBT en la pontificia universidad javeriana (tesis inédita de pregrado). Pontificia Universidad Javeriana, Bogotá, Colombia.

Ramírez, O. (2015). La comunidad LGBTI en Colombia: un estudio socio jurídico sobre la realidad del matrimonio (tesis inédita de pregrado). Universidad Católica de Colombia, Cundinamarca, Colombia.

Rodríguez, C. (2008). Logros y desafíos del movimiento LGBT de Bogotá para el reconocimiento de sus derechos una mirada desde la acción colectiva, las estructuras de oportunidad y la política cultural (tesis inédita de maestría/doctorado). Pontificia Universidad Javeriana, Bogotá, Colombia.

Rodríguez, L. (2017). Movimiento LGBTI en Colombia: un acercamiento al contexto histórico-político desde los acontecimientos de mayo del 68 y las revueltas de stanwall in que dieron origen al surgimiento del movimiento LGBTI en Colombia (tesis inédita de maestría/doctorado). Universidad de Cartagena. Cartagena de Indias, Colombia.

Rubin, G. (1986). El tráfico de mujeres: notas sobre la "economía política" del sexo. Nueva Antropología, VIII (30), 95-145.

Scott, J. (1996) El género: Una categoría útil para el análisis histórico. En: Lamas Marta Compiladora. El género: la construcción cultural de la diferencia sexual. PUEG, México. 265-302

Sosa-Sánchez, I. (2013). Aproximaciones teóricas sobre el género, la reproducción y la sexualidad. Revista de Ciencias Sociales de la Universidad Iberoamericana. VIII (15), 182-206.

Thurén, B. (1993): El poder generizado: El desarrollo de la antropología feminista. Madrid: Universidad Complutense.

Toro-Alfonso, J. (2012). El Estado Actual de la Investigación Sobre la Discriminación Sexual. Terapia psicológica, 30(2), 71-76. 
Turizo, J. Y Iglesias, M. (2010). Comunidad Lgbt: Historia y reconocimientos jurídicos. Revista Justicia, (17), 78-110.

Valdés, A. (1981). Historia y presente de la homosexualidad. Madrid: Akal editor.

Veiga, Y. (4 de mayo 2017). Los países que castigan la homosexualidad con pena de muerte. El correo. Recuperado de https://www.elcorreo.com/ bizkaia/sociedad/201705/04/paises-castiganhomosexualidad-pena-20170504175503.html
Velandia, M. (2007). Historia del movimiento homosexual colombiano desde sus orígenes hasta la culminación del siglo XX. Manuel Antonio Velandia Mora, Autografía y Artículos, 25.

Wittig, M. (1971). Las Guerrilleras. Barcelona: Seix Barral.

Young Iris (1992). Marxismo y feminismo, más allá del "matrimonio infeliz" (una crítica al sistema dual). El cielo por asalto, II (4), 41-56. 